\title{
Effects of procedural variables upon reversal and interdimensional shift performance: II'
}

PEdER J. JOHNSON, ANME FISHKIn AND LYLE E. BOURNE, JR.

UNIVERSITY OF COLORADO

College Ss were found to accomplish a reversal (R) shift faster than an interdimensional (IRD) only when simple preliminary instructions were used. The results suggest that with sufficient information Ss adopt a hypothesis-testing strategy, (in concept identification problem) similar to that described by Restle's model (1962).

Contrary to typical results (Kendler \& Kendler, 1962), Johnson (1965) found no difference in difficulty between $R$ and IRD shift in the solution of a simple concept identification (CI) problem. An examination of procedures used in various experiments suggested certain factors which might possibly affect the relative difficulty of $\mathrm{R}$ and IRD shifts. A subsequent study (Johnson et al, 1965), designed to assess two of these variables-method of stimulus presentation and presence of cue cardsfailed to explicate the discrepant findings. Still another difference between Johnson's experiment and some studies of solution shifts lies in the nature of instructions. Whereas in most experiments directions to $S$ were brief and vague, Johnson provided detailed information on the nature of the problem and its solution. These instructions may have induced a hypothesis-testing type of approach to the task. If Ss did select, test and reject alternative possible solutions-particularly in a manner suggested by recent hypothesis models of concept identification (Restle, 1962) - no performance difference between shifts would be expected. These models assume that Ss start over, i.e., resample at random from the complete population of hypotheses, with every error trial. It follows therefore that any shift in problem solution that results in an error trial will lead to the same number of errors in postshift performance. The present experiment investigated the effect of instructions on R-IRD differences, predicting the usual difference with simple instructions and no difference with detailed instructions.

Method

The Ss (48 undergraduates) were randomly assigned to the following conditions of a $2^{4}$ factorial design: Shift-R or IRD; Dimensions-2 or 4 irrelevant; Problem-number or form dimension relevant; and instructions-simple or detailed. In the simple instruction condition, $S$ was merely told that a series of patterns would appear on a screen before him and it was his task to respond by indicating the correct one of two categories for each pattern. Following each response $\mathrm{S}$ was told by $\mathrm{E}$ whether he had responded correctly. In the detailed instruction condition, Ss were also provided with a list of five possible di- mensions and told that the solution to the problem involved one of them. The $\mathrm{E}$ then showed $\mathrm{S}$ a sample solution, e.g., if size was the relevant dimension then all large patterns would go into one category and all small patterns would be assigned to the other category. In addition to the instruction variable this experiment differed from the previous experiment (Johnson et al, 1965) in two respects. Ten instead of 15 trials constituted criterion for both phases of the experiment and the stimuli were slides projected upon a translucent screen instead of cards presented directly to $\mathrm{S}$. In these respects it is similar to the Johnson (1965) experiment which found no R-IRD difference.

\section{Results and Discussion}

Since separate analyses on errors and trials to criterion on pre- and postshift performance showed essentially the same results only the error data are presented. The analysis of variance on the preshift data showed none of the mean affects or interactions to be reliable.

Analysis of variance on the postshift data showed dimensions, $F=13.83, \mathrm{df}=1 / 32, \mathrm{p}<.01$, Shift, $F=6.45$, $\mathrm{df}=1 / 32, \mathrm{p}<.05$, and Problem, $F=6.20, \mathrm{df}=1 / 32$, $p<.05$, all to be significant factors. The Dimension effect was the result of the usual increase in task difficulty with increased number of irrelevant dimensions. The significance of Problems resulted from the greater difficulty of number as opposed to form as a basis for sorting stimuli. The Shift effect can be seen in Fig. 1. which shows mean errors to criterion for $\mathbf{R}$ and IRD shifts with simple and detailed instructions combined over dimensions and problems. From Fig. 1 it can be seen that the IRD shift is generally more difficult, but that shift difficulty interacts with the type of instructions given. The analysis of variance

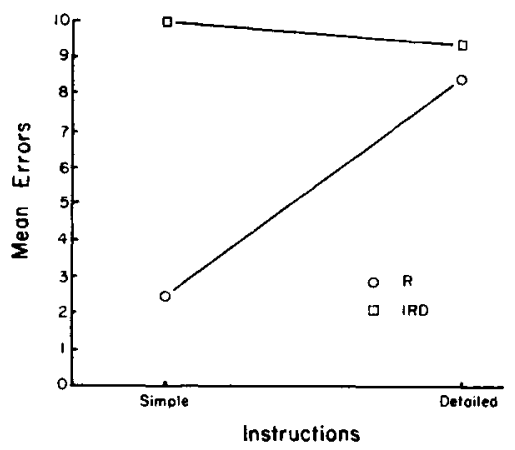

Fig. 1. Mean errors to criterion on postshift phase for $R$ and IRD shifts on Simple and Detailed instruction conditions. 
showed the Shift by Instruction interaction to be only marginally significant, $F=3.42$, df $=1 / 32, p<.10$. Such an analysis, however, does not provide an explicit test of the hypothesis that there is no R-IRD difference under the detailed instruction condition, but a significant difference under the simple instruction condition. Planned pairwise comparisons showed no reliable difference between $R$ and IRD shifts for the detailed instructional condition, $F=.20, \mathrm{df}=1 / 32$, while the $R$ shift was significantly less difficult, $F=9.85, \mathrm{df}=1 / 32$, $\mathrm{p}<2.01$, for the simple instructional condition.

These results suggest that the added information given $S$ s with detailed instructions was responsible for the disappearance of any R-IRD difference in Johnson's (1965) experiment. Instructions which include explicit labels for the stimulus dimensions, which indicate that only one dimension will be relevant, and which demonstrate a possible solution to such a problem, all probably combine to induce a hypothesis-testing type of responding. Under these circumstances the expecta- tion of no difference between $R$ and IRD shifts based on a literal interpretation of hypothesis testing theories (Restle, 1962) is realized.

\section{References}

Johnson, P. J. Some factors affecting solution of transfer in concept identification problems. Ph.D. Thesis, University of Colorado, 1965.

Johnson, P. J., Fishkin, Anne, \& Bourne, L. E., Jr. Effects of procedural variables upon reversal and interdimensional shift performance: I. Psychon. Sci, 1965, 4, 63-64.

Kendler, H. H., \& Kendler, T, S. Vertical and horizontal processes in problem solving. Psychol. Rev., 1962, 69, 1-16.

Restle, F. Selection of strategies in cue learning. Psychol. Rev., $1962,69,329-343$.

\section{Note}

1. This research was undertaken in the Behavior Research Laboratory, Institute of Behavioral Science, University of Colorado and is Publication 62 of the Institute. The work was supported by Grant MH 08315 from the National Institute of Mental Health, U. S. Public Health Service and by Grant GB 3404 from the National Science Foundation. 